\title{
Cross-cultural Mediation in ELF Migration Contexts: Pedagogical Implications on ELT Multilingual Settings
} Silvia Sperti a * (D)

\author{
a Roma Tre University, Via Ostiense 234-236, Rome 00164, Italy
}

\author{
Received 16 April 2019 | Received in revised form 15 July 2019 | Accepted 30 July 2019
}

\section{APA Citation:}

Sperti, S. (2019). Cross-cultural mediation in ELF migration contexts: Pedagogical implications on ELT multilingual settings. Eurasian Journal of Applied Linguistics, 5(2), 269-286. Doi: 10.32601/ejal.599253

\begin{abstract}
ELF cross-cultural interactions and mediation processes in specialized migration settings are often characterized by 'gatekeeping' asymmetries between the participants involved challenging a successful meaning negotiation (Guido, 2008). The exploration of migration encounters (Sperti, 2017) is particularly useful in the analysis of naturally occurring dialogues among ELF users, since it shows how ELF speakers, engaged in intercultural interactions, appropriate the English language according to their own native linguacultural and paralinguistic schemata, and to specific pragmalinguistic purposes and processes. The multimodal investigation of the occurring hybridization processes is focused on (i) ELF redefinition of existing native paralinguistic correlates in the pragmalinguistic use of an ELF variation; and (ii) resulting L1 transfers affecting the performing of speech acts and the conversational composition and progress. The analysis reveals (a) the mediation of meaning, experience and intentionality in terms of resulting lexical, syntactical, and register performance; and (b) the role played by prosody and paralanguage in the mutual acceptance of speakers' intentions, attitudes, and cognitive schemata, in spoken specialized discourse related to medical and legal integration, mediated migration narratives, cross-cultural conceptual representations and reception of traumatic experience. The heuristic approach applied to the analysis of data derived from the exploration of real plurilingual cross-cultural exchanges is particularly useful in the promotion of the conscious use of cross-cutting strategies as powerful learning tools embedded in the language learning process, with the ultimate aim of (i) investigating the possible impact of migration on teacher education, (ii) defining an ELF-aware pedagogical framework in plurilingual educational settings, and (iii) enhancing the development of learners' skills in intercultural communication.
\end{abstract}

(C) 2019 EJAL \& the Authors. Published by Eurasian Journal of Applied Linguistics (EJAL). This is an open-access article distributed under the terms and conditions of the Creative Commons Attribution license (CC BY-NC-ND) (http://creativecommons.org/licenses/by-nc-nd/4.0/).

Keywords: ELT; migration; English as a Lingua Franca; language mediation; intercultural communication.

\footnotetext{
* Corresponding author. Tel.: +39-349-2189129

E-mail address: silvia.sperti@uniroma3.it
} 


\section{Introduction}

\subsection{English as a Lingua Franca and migration contexts}

The spread of English as a global lingua franca and the attention to the related socio-linguistic phenomena is unquestionable as confirmed by the large amount of research about ELF in the last decades. As claimed by Mauranen (2018, p. 8), ELF is "English is spoken in situations with widely varying combinations of participants, including first-language speakers of different varieties"; In other words, it may be defined as "a contact language between speakers or speaker groups when at least one of them uses it as a second language" (Mauranen, 2018, p. 8). Seen from this perspective, the use of English as a shared common language is particularly frequent in migration contexts where speakers from different socio-cultural and pragmalinguistic backgrounds exchange meaning for communicative purposes.

The multilingual settings in this paper may be defined as migration domains involving Western experts - namely legal advisors or officers, and mediators - and non-Western migrants (asylum-seekers and refugees included) interacting in professional contexts. It will be argued that in such communicative conditions ELF is used in terms of variations developed from speakers' L1 structures transferred onto the use of English. In other terms, ELF users act according to a process of authentication (Widdowson, 1979), shaping the English language by means of and according to their native pragmalinguistic conventions. The pragmalinguistic investigation of such interactions allows (i) to detect processes and forms of appropriation across different socio-cultural backgrounds, and (ii) to define power/status asymmetries emerging in ELF encounters, at times ending in communication failure or miscommunication (Guido, 2008).

The emerging new linguistic landscapes in English Language Teaching (henceforth, ELT) reveal the importance of considering the current societal changes and their impact on education in defining innovative approaches and trends. In this sense, the heterogenous scenario appearing from migration encounters is particularly indicative of the impact that multilinguals and their multicultural backgrounds may have on language teaching as well as teacher education nowadays.

\section{Theoretical background}

\subsection{Defining mediation}

In the last decades mediation has been defined and formulated variously in different fields of scientific research, from a socio-cultural and anthropological perspective where "mediation is the process through which humans deploy culturally constructed artifacts, concepts, and activities to regulate (i.e. gain voluntary control over and transform) the material world or their own and each other's social and mental activity" (Lantolf \& Thorne, 2006, p.79) to a sociolinguistic approach in which, 
"[The intercultural mediator is someone who could] operate their linguistic competence and their sociolinguistic awareness of the relationship between language and culture and the context in which it is used, in order to manage interaction across cultural boundaries, to anticipate misunderstandings caused by difference in values, meanings and beliefs, and thirdly, to cope with the affective as well as cognitive demands of engagement with otherness" (Byram, 1995, p.25).

Mediation has acquired paramount importance in the 2001 Common European Framework of Reference - and more recently in the CEFR latest edition (2018) - and in language policies where mediation is "central to the teaching-learning process, not only in the context of teacher-learner interaction but also in that of interaction between learners or between teaching materials and learners" (Council of Europe, 2010, pp 11-12). In this pedagogical perspective, mediation "involves a constellation of teachers' conceptual frames, practices and ways of being that are at the play in any given moment and which are interwoven over time in the act of language teaching" (Kohler, 2015, p.193).

Since negotiation processes are typical of international and intercultural communication. mediation has been explored in different ELF communicative settings, from the academic and professional interactions to the migration contexts where ELF users typically establish mutual intelligibility and "mediation is successful if the parties to the mediation achieve mutual understanding, that is, if the intermediary is able to help the others achieve their communicative goals" (Hynninen, 2011, p. 972). Excessive use of quotations in this and previous two paragraphs.

In this conceptual perspective, authentic processes of cross-cultural mediation in migration contexts are here explored focusing on the multimodal dimensions of crosscultural legal-bureaucratic and asylum-seeking spoken interactions, characterized by the employment of ELF variations. Multimodality in this paper is meant as the negotiation of meaning through the simultaneous action of different linguistic levels namely linguistic, paralinguistic and extralinguistic cues - enacted by ELF users. Indeed, speakers involved reveal the use of different strategies of appropriation of the English language according to L1 linguacultural schemata and pragmalinguistic processes. In these multifaceted interactional dimensions, very often 'gatekeeping' and status asymmetries (Guido, 2008) among the participants in interactions influence the natural exchange of illocutionary intentions (meant as the speaker's attitudes and meaning conveyed) and perlocutionary effects (meant as the receiver's responses and reactions). The exchange of speech acts affects the whole communicative process from speakers' prosodic strategies actualized in speech segmentation and acoustic variations (in terms of voice quality, intensity, pauses and intonational strategies) to register and conversational dynamics (Sinclair \& Coulthard, 1975).

Various theoretical perspectives and assumptions sustain and justify the rationale of the current research objectives, i. e. (i) 'gatekeeping' asymmetries between the participants in interactions occurring in immigration domains, where communication 
is often characterized by challenging pragmalinguistic accommodation strategies and cross-cultural miscommunication (Guido, 2008; Roberts \& Sayers, 1987; Sperti, 2017); (ii) the theory of speech acts with a special focus on intentionality (Searle, 1969; 1983) conveyed by the speakers through the adoption of prosodic strategies of speech segmentation and acoustic variations (Nespor \& Vogel 1986; Selkirk, 1984); (iii) the interface between the multimodal construction of meaning and its perlocutionary effects on receivers from different socio-cultural and linguistic backgrounds in ELF intercultural interactions (Seidlhofer, 2011).

Such theoretical grounds provide the basis of the research objectives aimed at enquiring into the pedagogical implications of introducing authentic materials derived from ELF speakers' cross-cultural exchanges in migration contexts in the ELT (English Language Teaching) classroom. In this sense, the exploration of ELF users' attitudes in the construction of meaning and understanding in cross-cultural mediation through phonopragmatic strategies (Sperti, 2017) is particularly useful in considering the negotiation of speakers' attitudes, emotions, and socio-cultural 'schemata' (meant as an interaction of L1 background knowledge and socio-pragmatic behaviours shared by a speech community (Carrell, 1983) in intercultural communication. At the same time, language learners - in secondary education as well as in undergraduate and postgraduate courses - become aware of the miscommunication and communication breakdown resulting from status asymmetries in unequal encounters during cross-cultural mediation processes as well as daily spoken interactions in a multilingual and multicultural communicative dimension.

This is particularly relevant when insights from the new descriptors in the CEFR are taken into account: in the 2018 Companion Volume to the CEFR for Languages, new scales for mediation are introduced with the aim of promoting more integrated, cooperative and collaborative classroom tasks and the implementation of crosslinguistic mediation and the development of plurilingual/pluricultural competence among language learners.

The exploration of the following data, derived from the ethnographic research of migration and its impact on language use, aims at defining practices and strategies for the introduction of authentic materials in the training of mediation - in its multiple aspects and interdisciplinary facets - within the language classroom.

\section{Method}

\subsection{Investigating ELF mediation processes}

The multimodal research approach applied here to migration contexts and domains aims at a pragmatic-oriented linguistic and paralinguistic investigation of ELF speakers' communicative behaviours - naturally aimed to realize illocutionary acts and to produce perlocutionary effects in listeners - in cross-cultural oral interactions. The tangled connection between prosody and pragmatics, actualized in linguistic outputs, underlies the research assumptions and approaches to the analysis of cross- 
cultural communicative settings. In other words, illocutionary acts and perlocutionary effects are affected by a culture-oriented discourse construction derived from L1 interferences that interactants mutually actualize in conversation, at any stage and in any pragmalinguistic dimension. These peculiar dimensions of ELF interactions are especially relevant and emerging in mediation contexts where meaning should be not only conveyed but also properly negotiated.

Thus, the main objective of this investigating approach is to explore (i) how speakers' suprasegmental and paralinguistic features (prosodic strategies and uses such as pauses, volume, speech rate) are influenced by underlying pragmatic aims and how they affect the mutual exchange of speech acts (i.e., actions performed through sentences) in conversational interactions and their resulting perception and interpretation. Moreover, the investigation is aimed at exploring (ii) how native syntactic and stylistic patterns are transferred to the use of different ELF variations and to which extent they impact on the phonopragmatic production and perception of the English messages transmitted in intercultural encounters and, as a consequence, improve or hinder the cross-cultural mediation process.

The following extracts, therefore, may be explored under various perspectives: first of all, the phonopragmatic analysis accounts for different acoustic and prosodic parameters, such as pitch frequency, pitch contour, speech rate, vowel and tonic syllables duration, pause duration at phrase boundaries and acoustic intensity. The multimodal investigation further reveals ELF register and conversational dynamics which are at the basis of specific ongoing pragmalinguistic fulfilment or gaps in communication.

Data here represent naturally occurring and real exchanges in an ever-changing world where diverse individuals, lives and experiences collide and negotiate mutual representations, intentionality and attitudes, by means of expanding, creative and easily exploited mediation strategies involving ELF variations.

The considerable relevance of the use of authentic materials ${ }^{\dagger}$ in ELT and language teaching in general, is particularly important in the training not only of future mediators and operators for international and intercultural communication, but of language learners at any stage of their secondary education. Deductions in an ethnographic research conducted by means of data-driven methodology are particularly useful for projecting teaching activities and tasks based on the exploration of prosodic and paralinguistic features of spontaneous speech in intercultural exchanges $\ddagger$

\footnotetext{
$\dagger$ The following data were recorded in completely unconstrained, spontaneous and natural conditions, but, obviously, they have also been collected in a manner that preserves and safeguards the privacy of both participants and non-participants - aspect which, especially in workplaces involving refugees and asylum-seekers, is particularly important. Despite the privacy constraints, data allow for an extensive scientific investigation of different types of inferences that have emerged in turn from the analysis.

₹ The following data have been classified and analysed according to a scheme established to preserve as much information as possible and allow inferences from conversations between participants. To protect the privacy of any interactant who came within the range of the microphone and whose acoustic information is saved and represent intelligible speech, proper nouns, places, cities, and villages which may be easily recognized, thus revealing precise
} 
The following data are part of a larger corpus of interactions recorded in a centre for legal advice for asylum-seekers and refugees in South Italy during a two-year period of fieldwork (Sperti, 2017). The participants in the following extracts are (i) legal advisors (LA), all Italian native speakers, adult learners of English with fairly basic linguistic competence; (ii) asylum-seekers and migrants (AS), male and female African citizens with varied linguistic competence of English (some of them are native speakers of Hausa, Igbo, Yoruba, Ewe, Twi (all Niger-Congo languages) and Arabic, as well as ESL speakers (actually they consider English as their native language) and therefore are very competent. Other speakers are illiterate and employ an ELF variations to communicate with their own fellow country-men and -women and with Italian people. Most part of ASs are Italian as a Lingua Franca (ILF) users and possess a basic knowledge of the Italian language, particularly influenced by the local and regional linguistic and suprasegmental features of the Italian variety spoken in the area where they live and work for an indefinite period of time; (iii) language mediators (IM), Italian and Serbo-Croatian speakers and all graduates or postgraduates in foreign languages. Their register proficiency of English is often academic but, in some cases limited, to basic levels of competence§.

This assorted linguistic background as a starting point for investigating mediation dynamics is particularly interesting as it is indicative of the ongoing variety of approaches and attitudes in the use of the English language by non-native speakers worldwide.

The following transcriptions are presented through a notation applied from Edward's (1997) system which can be summarized in the following table:

Table 1. Transcription notation adapted from Edward's (1997) system

\begin{tabular}{ll}
\hline$[$ ] & $\begin{array}{l}\text { Square brackets mark the start and end of overlapping } \\
\text { speech }\end{array}$ \\
underlining in black & Prominence associated to pitch accent \\
CAPITALS & Louder speech \\
$\circ \circ$ & Raised circles enclose quieter speech \\
$(.)$. & Pauses \\
$()$. & Micropauses \\
$::$ & Vowel elongation; the more colons the more lengthening \\
hhh & Aspiration \\
$><$ & Speeded-up talk \\
$<>$ & Slowed-down talk \\
$=$ & Immediate "latching" and turn-taking
\end{tabular}

information about the identity of any participant, have been concealed and signalled in the text with asterisks (i.e. four $* * *$ for places, five $* * * *$ for names).

$\S$ It is relevant to notice that in a typical intercultural encounter, involving specialized settings, an operator, a migrant (asylum-seeker, refugee or international protection holder) and an intercultural mediator are seated together. Data will show however that in most cases this is still a theoretical perspective in considering mediation in migration contexts while in practice this kind of encounter often occur in irregular communicative settings and modalities. 
The audio recordings have been acoustically screened and transcribed with the aim of emphasizing the emerging linguistic and paralinguistic parameters:

- Phonological and extralinguistic features (signalled in the transcriptions with bold green, capitals and black underlining);

- The use of modality and verbal choices (signalled in the transcriptions with bold blue);

- Key-textual structures (signalled in the transcriptions with bold pink);

- Stylistic tendencies (signalled in the transcriptions with bold brown);

- L1 interferences (signalled in the transcriptions with italics);

- ELF accommodation strategies and code-mixing (signalled in the transcription with bold red for single lexical items and red underlining for ELF syntactical clusters).

\section{Results}

\subsection{Mediation in context: insights from cross-cultural migration encounters}

The following extracts aim at exemplifying how authentic ELF materials may be used in ELT at different level of linguistic investigation and observation, impacting on learners' and teachers' awareness of new multilingual and multicultural communicative scenarios. More specifically, the multimodal investigation of the hybridization processes - meant as accommodative lexico-grammar processes, typical of ELF interactions - is focused on:

(i) the redefinition of existing native paralinguistic correlates in the pragmalinguistic use of an ELF variation;

(ii) resulting L1 transfers affecting the performing of speech acts and the conversational composition and progress;

(iii) the mediation of meaning, experience and intentionality in terms of resulting lexical, syntactical, and register performance;

(iv) the role played by prosody and paralanguage in the mutual negotiation of speakers' intentions, attitudes, and cognitive 'schemata' in spoken interactions.

In this perspective, the linguistic analysis of ELF mediation processes may reveal all the pedagogical implications for teachers as well as for learners: the employment of authentic materials in the ELT classroom may be integrated to a tailor-made application of innovative approaches to the traditional teaching practices.

\subsection{Paralinguistic correlates of the pragmalinguistic use of an ELF variation}

In the following passages, different socio-cultural 'schemata' about migration and asylum experience emerge from the participants' conversational exchanges. Such interactions, here exemplified by means of short extracts, may be adapted as teaching materials in the ELT classroom to the extent that they are properly introduced in 
terms of socio-cultural setting and sociolinguistic behaviour. In this sense, teacher education is also fundamental in providing teachers with tools and trajectories triggering a reflection on their own teaching context and the possibility to introduce content-rich materials and new activities in classroom.

Consequently, the following examples and their linguistic analysis are meant to give insight into real mediation processes in migration contexts with the aim of promoting the exploitation of authentic materials in the projecting of mediation activities in the ELT classroom in an ELF-aware perspective. The register and conversational analysis of ELF exchange provide additional cues for the preparation of teaching activities based on authentic materials such as role-plays, project works, action-oriented exercises and reflective tasks.

For instance, what follows is an extract taken from a conversation between an Italian legal advisor and a Nigerian asylum-seeker. In (1) the Italian conative question tag no? affects the ELF pragmalinguistic dimension of LA's conversational management till the AS's cue of misunderstanding with the request of IM's intervention (i.e. please can you interpret this?):

(1) LA: Your permit to stay is sussidiaran protection (.) no?

$A S: \mathrm{Mmm}$

$L A$ : Is write on permit (.) no?

$A S$ : Yeah

$L A$ : Have you here your permit?

$A S$ : Yeah

$L A$ : Can you show me (.) please?

$A S: \mathrm{Mmm}$

$L A:$ Ok (..)

$A S:$ Scusa (..) please can you interpret this?

IM: Yeah

$A S:$ Ok (.) go ahead

In (2), the AS's latching signals his pragmalinguistic attitude towards IM's series of Western-oriented questions aimed at inquiring into his past experience, in the attempt to take her conversational turn:

(2) IM: Where did you=

$A S:=I$ don't remember the dates but I can remember what happened (.) and there e::h $[\mathrm{mmm}(.) \mathrm{mmm}] \mathrm{I}$ just (..).

In (3), AS's use of repeated summoning and supporting moves about his court appeal is strictly related to his phonopragmatic use of micropauses and rising intonational patterns: 
(3) AS: In questura finish (.) but now they say to find one lawyer (.) I call my lawyer in $* * * *$ and my lawyer said I should reappeal (.) he said I should reappeal so that if I can reappeal they will give me back my document

$L A: M m m($.) but have you some paper about your reappeal?

$A S$ : Mmm? What? I want to reappeal (.) the lawyer said I should come and e::h call the lawyer in $* * *$.

The repetition of the same request affects the ELF structure of the exchange where different illocutionary goals are realized: the AS's need for help and the LA's codifying of legal events.

In (4), the move exchanges between LA and AS are particularly interesting: LA variously reformulates her questions in order to obtain a certain reply from the AS who focuses his attention on time (before, the time before) rather than on result (as in LA's use of present perfect, i.e. *have you speak?, *have you spoke?):

(4) LA: Ok (.) so I want to this (.) have you speak with the commission or not?

$A S$ : Commission?

$L A$ : Commission is an organ who decide about your asylum request (..) then make you (..) a lot of question about the reason why you decide to left your country ehm

$A S$ : Yes (..) before (.) before

$L A$ : Have you spoke with them?

$A S$ : Yes I spoke (.) I spoke with them (.) the time before.

\subsection{L1 transfers on ELF conversational patterns}

In intercultural exchanges involving mediation practices in migration contexts, socio-cultural schemata and conceptual L1 transfers easily emerge in the conversational dimension as, for example, in (5) where the L1 cultural concept of 'banishing' is transferred onto the ELF pragmalinguistic construction of meaning:

(5) AS: The other relatives (.) you have to accept it I believe in $* * * * *$ so when you don't accept the religion you will be banished

$I M$ : Now or before? When you was a child was the same?

$A S$ : Yeah it was the same (.) when I was a child my parents were banished

(.) do you understand? So they were banished at all from the community.

And in (6) when the IM tries to collect useful information about the AS's personal background justifying her probing questions (not for me ok?) and connecting verbal patterns to corresponding epistemic perspectives (see, know, think):

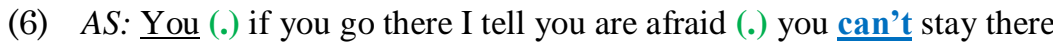
IM: Mmm why? 


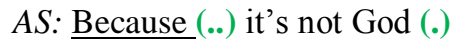

$I M$ : It's important for the commission ok? [yes, yes] Not for me ok?

$A S$ : Is bad religion (.) I want to live a good life (.) I don't want to kill the people::

$I M$ : Because they kill the people?

$A S$ : Yeah (..) I told you that

$I M$ : You see? You know? Or you think?

The same L1 conversational transfers emerge in the management of Westernoriented interrogative structures, as in (7):

(7) AS: Yeah (..) maybe if I can get a work or maybe school (.) maybe I can get a work so that

$I M:$ Mmm (.) yes but we need to understand (..) ok (..) but do you have contact with your family? Do you have now contact with them?

$A S:$ Mmm (..) I have but before I came I left my document (.) the numbers

$I M$ : Ah ok (.) so you don't call them

IM's linguistic and paralinguistic effort is devoted to fulfil her illocutionary goals, i.e., eliciting new information from the AS and finally persuading him to accept her suggestions, yet neglecting the cross-cultural gap between her Western perspective in considering personal values and social assistance and his non-Western schemata (e.g. about the sense of family and community), which probably a language mediator would have been able to fill.

On the other hand, in (8) LA claims her opinion about the AS's legal status through a declarative which yet is not properly perceived by the migrant who is asking for asylum:

(8) LA: We didn't give this to the police (.) because is better I think to (.) prepare the story for the commission (.) but (.) before to give (.) to the police or commission (.) is better that we read very well (.) is better to see it before $[\ldots]$

$A S:$ Ehmm (..)

$L A$ : Do you remember when you have to fill in the $* * * *($.$) the paper with$ the police (.) do you remember? With the police (..) do you have a copy?

$A S:$ No (.) I don't remember the date

$L A$ : Not the date (.) we want to know if in the end of the interview with the police they gave you a copy of that interview

The lawyer, indeed as an ELF user, tends to transfer her L1 phono-prosodic features to spoken interactions: she operates evident L1 variations involving intonation (patterns of pitch rises and falls and pattern of stress), contrastive stress (used to mark words, phrases or clauses), pauses (used to signal pragma-syntactic boundaries), speech and articulation rate, intensity, distribution of theme vs. rheme information in intonation units, all paralinguistic and phonopragmatic correlates typical of her Italian variety. 


\subsection{Pragmalinguistic mediation of meaning and ELF register performance}

In migration contexts the specialized dimension on discourse is often characterized by the use of legal and technical vocabulary as well as ELF accommodation strategies affecting the use of register.

This is what may be noticed in (9) where the Nigerian AS explains his opinion about European legal concepts and international laws through a metalinguistic reflection:

(9) AS: Mmm (.) the problem is that I just don't need more explanation about this (..) so that you know you know the law more than me so that I need my explanation in in European laws is different from African law I know that not all of yours know all the laws but if you know them better all the laws so I need explanation about this ok about the borders about how to get the border how to (.) you know cause I know that of the Italian law not everyone of you know the Italian law because of the language variation we find it difficult to know the law what if you can help to me to how to understand the law is what the law said (.) what the law say so I can understand more better (..) because when I came in this city what I didn't know of (..) I don't (..) I haven't been no more expected about the law (..) European laws and Italians law (..) are you understanding what I'm saying.

The employment of conative contacts (you know) and coherent/cohesive devices (the problem is that, so, because) signal his ELF mediation of meaning and concepts by means of stylistic and register resources.

In (10), the LA adapts her register repertoire in s segment of speech is an atypical intercultural mediation process based on persuasive aims and pragmalinguistic power asymmetry and performed without the mediator's assistance:

(10) $L A$ : Ah (.) so (.) I explain tha that the situa::tion is difficult (.) because (.) you asked asylu::m (.) the::n commission decide for a negative answer (..) and the:::n you make an appeal but the appeal (..) ehh have a negative answer (.) and ${ }^{\circ}$ so they give you this paper where is $\underline{w r i t e}^{\circ}($.$) that you$ have ten days from this date

$A S$ : To give the contract

$L A$ : No to give the contract (.) to write something to change this situation (.) because if after ten days (.) you have not (..) other reason (.) they have to give you an expulsion (..) this is the normal procedure in our system (..).

\subsection{Prosody and paralanguage in the spoken discourse of migration}

In other cases, mediation in migration contexts is strictly related to specialized discourse, such as medical and legal domains of the socio-economic integration 
process, where it is usual to get involved in mediating migration narratives, crosscultural conceptual representations and reception of traumatic experience.

In (11), for example, the LA employs her linguistic and paralinguistic properties to introduce legal and bureaucratic procedure related to AS's rejection of asylum application:

(11) $L A$ : You must prepare also a declaration (.) because now e::h police ask to prepare like a declaration where you say "it's impossible for me to obtain passport from Nigeria embassy because we are refugee (.) we have some problem in other country so we want to (.) we don't like to have any contact with the embassy".

The ELF use of deontic modality (you must) and of pleonastic personal deictics (we) may be interpreted in the 'gate-keeping' structural frame usually established in professional workplaces involved with migration and asylum-seeking.

In (12) again the LA mutually exchanges with her interlocutor concepts and ideas mediating through discourse strategies:

(12) $A S$ : On Thursday

$L A$ : Friday (.) in the afternoon (.) I will call again $* * * *$ because yesterday he said that maybe in Friday afternoon is possible to make this interview

$A S$ : Ok (.) I think that at the end of this month everything is finished

$L A$ : Yes (.) I know (.) we try to resolve this problem about permit (.) ok? Then the situation is not easy (.) the permit cannot resolve every problem but ehm is the first step (.) ok?

In the selected extract (as well as in the whole exchange) the lawyer employs phonopragmatic and pragmalinguistic strategies to be more effective and persuasive as she tries to convey her illocutionary intents also through paralinguistic (e.g. ok?) and linguistic means (e.g., the situation is not easy, we try to resolve, maybe, I think, I know).

In (13) a very long speech is mediated by an IM under the LA's Italian indications:

(13) $I M$ : We are here all together in order to (..) because we have to inform you about very important news (.) legal news (.) ehm these new are just (..) was made just some days ago from our government and are very important news about this law which is for all the people who was denied from the commission (.) received a negative answer (.) this law is for all the people who has a negative answer and these people can ask to have a new permit to stay which probably can be for humanitarian reason (.) but is not written inside this document (.) probably can be this kind of (.) for humanitarian reason (.) so (.) for all these people who had negative answer [...] we now we can fill up together this application form in order to make this kind of document (.) now we can arrange to fill up this application form (.) ehm we can arrange to have a time (..) or a calendar and timetable in order to meet you and fill up this 
application form (.) which can be given to the police (.) [...] ehm when we finish to work in order to fill up all the application form we can go for all of you (.) for all the application form to the police station (.) so don't worry about for example if somebody can ehm get this application form before you (.) because now the important is to fill up for everybody and after we can give this to the police station (.) [...] in this application form (.) ehm you can write if you want (.) you decide to be ehm listened by the commission (.) again (.) ehm but probably if you decide to be again in front of the commission this period could be longer than to get this permit to stay (.) so probably can be better to decide not to go in front of the commission again (.) because in this way you can receive before this document (.) this permit to stay (.) ok?

To fulfil LA's illocutionary goals, the mediator activates different phono-prosodic strategies as revealed by the acoustic analysis: a wide variety of prosodic resources are employed to focus on lexical and semantic items with a pragmatic aim (such as pitch accent placement, phrase boundary placement, and focus marking, all signalled in the transcription). Here the mediation is taking place towards a group of migrants in a reception centre. The speech is a monologue with informative purposes: the legal content is mediated through the use of specific vocabulary (e.g. legal news, government, commission, permit to stay, humanitarian reason, application form); stylistic and register features with pragmalinguistic force (e.g. we, all together, very important news, the important is); specific verbal structures and modalized hedges (can, could, want, decide).

\subsection{Investigating ELF mediation encounters: insights and implications}

A multimodal investigation of mediation in migration contexts and cross-cultural spoken interactions in professional settings reveals above all L1 phonological patterns transferred to the use of the ELF variations by speakers belonging to different speechcommunities. On the other hand, communication breakdowns and misunderstandings may occur not only for linguistic gaps or inaccuracy, but also for a misinterpretation of socio-pragmatic use of paralinguistic correlates, such as silence or backchannels which may have culture-specific pragmatic and communicative effects carrying meaning about attitudes and feelings. Therefore, misunderstandings may arise whenever silence or other paralinguistic and prosodic cues are not properly interpreted by listeners belonging to speech communities that do not share the same communicative usage.

Moreover, ELF users' variations (marked - as seen - by a number of linguistic and paralinguistic transfers) are often employed with the aim of enabling and simplifying the accessibility of persuasive messages about crucial issues which are noticeably problematic for migrants and asylum-seekers. The conversational pattern often reveals and confirms the unequal biases typical of migration settings: the legal advisor's illocutionary force - actualized by means of declaratives and directives - vs. 
the migrant's perlocutionary effects of expressives and affectives of discomfort and communication failure.

By cross-checking phono-prosodic cues with lexical choices, it is evident that the participants employ different prosodic and intonational patterns to stress certain linguistic items. This is consistent with the adoption of syntactic and stylistic strategies, such as deontic modality, the use of aspect variations, code mixing and switching, and phatic contact; ELF syntactic and structural gaps, fillers and disfluencies as well as paralinguistic elements involving kinesics, proxemics and voice quality.

The intercultural encounters are here ongoing mediation processes: on the one hand, the LAs' and IMs' main objective is to provide migrants and asylum seekers with a better accessibility to legal and textual issues regarding the long and complex Western asylum-seeking procedure, which are different and partially unavailable, from the asylum-seekers' socio-linguistic and cultural backgrounds, or even conceptual repertoire. Yet, in some cases the Western speakers' willingness to fulfil their illocutionary goals of persuading results in an imposition of their perspective on the one side, and very often in miscommunication and bewilderment on the AS's hand.

The previous comments and insights are aimed at drawing attention to the multiple advantages and sources of inspiration which may be derived from the linguistic investigation of ELF spoken interactions in cross-cultural migration contexts for teaching purposes.

\section{Discussion}

\subsection{Introducing mediation in the ELT: authenticity and communicative skills}

The previous illustration of the considerable linguistic observations and comments which may be derived from real and spontaneous cross-cultural interactions in ELF mediation settings, demonstrates the extent to which authentic materials may be of special and powerful relevance in terms of pedagogical exploitation.

The analysis of real exchanges in professional mediation contexts is particularly useful not only to identify some significant aspects of the different linguistic and paralinguistic strategies adopted by the participants involved in migration settings this sentence should be finished. The speakers engaged in the previous mediation encounters are asylum-seekers, legal advisors and mediators who differently apply, onto ELF variations, prosodic tools according to culture-bound pragmalinguistic strategies and consequently conveying different effects on the receivers. These aspects and peculiarities are extremely useful in terms of teacher education as well as language acquisition and language teaching

On the one hand, in ELT it is of paramount importance the reflection on the significant role assumed by prosody on the use of tense and aspect, modality, 
simplification of terminology, legal-bureaucratic lexis and hedging. This approach emphasizes the importance of detecting illocutionary cues in real cross-cultural mediation encounters regarding asylum-seeking and traumatic experiences realized through ELF variations.

On the other hand, it is advisable that the projecting and realization of ELF-aware teaching materials are based on the actualization of: (i) lexical features (in terms of paraphrasing, simplification of terminology, accommodation strategies); (ii) syntactical features (such as the use epistemic and deontic modality and use of different verbal aspects); (iii) stylistic features (such as hedging and phatic contact used to maintain and value the receiver's attention, and verify the contact with the addressee; and rhetorical figures).

First of all, the pedagogical implications of adopting real mediation processes in classroom activities are related to the exploration of mediators' communicative effort for real and specific purposes, which involves a certain amount of suprasegmental and rhythmic features, appropriate for his/her interlocutors, and other paralinguistic and extralinguistic features (voice quality, facial expressions, posture, gestures, eye movements and gaze, body movements and space management). Since cross-cultural mediation exchanges are spontaneous and urgent, they also show greater emotional involvement in the topic of discourse or in the interaction which may emerge in different ways as speakers modify and affect their speech prosody according to personal linguacultural transfers from L1, as well as pragmatic conveyance of intentionality or culture-bound experience or 'schemata'.

Language learners are, therefore, involved in reflective activities involving metalinguistic analysis of linguistic and paralinguistic occurrences in ELF settings. This use of authentic materials promotes the learner's awareness of receptive difficulties, if not misunderstandings, for any speaker involved in intercultural conversations, especially when different ELF variations are spoken as a means of communication with low level of proficiency and accuracy and by speakers whose native languages possess intonational systems which considerably differ from each other.

Moreover, the new descriptor scales for mediation emphasize the focus on teaching actions aimed at relating learning goals to real-world language use, within a pedagogical framework based on action-oriented learning. Mediation is seen as a tool for enhancing the role of learners as social agents. In teaching practice this implies involving them in the learning process through the extensive use of tasks and materials which enable learners to use all their linguistic resources, their crosscutting skills and their intercultural competences.

More precisely, in the CEFR new scales are provided for mediating a text, for mediating concepts, for mediating communication, as well as for the related mediation strategies and plurilingual/pluricultural competences. In these terms, the previous authentic exchanges and the series of pragmalinguistic aspects emerged from the 
multimodal analysis may reveal a considerable potential in training new skills and new strategies as required by the CEFR in language teaching and learning.

As for mediating a text, the listening and reading of ELF variations in real intercultural encounters imply the training of skills in processing and translating written and spoken language activating the learners' personal criticism towards the creative use of English as a global language.

As for mediating concepts, migration exchanges are particularly useful in the classroom to encourage and support learners' skills and strategies to cooperatively negotiate meaning and manage interaction through an integrated analysis of key concepts and strategies applied by speakers involved in the conversation, with a relevant impact on the well-being of the increasingly multicultural classroom.

As for mediating communication, analyzing and enacting cross-cultural exchanges set in ELF migrant or pluricultural contexts promote the learners' awareness of pluricultural space where they act as intermediaries facilitating communication in delicate situations or facing and solving interactional conflicts.

Therefore, the follow-up of this research should be a deeper investigation of the effects produced by the exploration of ELF authenticity, emerging from mediation exchanges, onto language learners in managing different aspects of mediation. Training learners for the analysis of the meaning negotiation in plurilingual and multicultural dimensions, the mediation of a cross-cultural exchange, and the reformulation of a text may be useful for the exploration of the perlocutionary effects and potential triggers for misunderstandings, derived from all the participants involved in these kinds of cross-cultural interactions. In this sense, therefore, the pedagogical implications of an ELF-oriented introduction of mediation concepts may be useful - at school as well as in academic contexts - not only to measure and detect the employment of phono-prosodic strategies revealing speakers' illocutionary acts, but also to make future mediators (responsible for difficult and embarrassing intercultural transactions) as well as language users aware of (i) the mechanisms underlying mutual positioning and perception, and (ii) possible vulnerabilities for misinterpretation, in order to avoid and prevent them.

\section{Conclusions}

The extracts from mediation processes in migration domains have revealed their special usefulness from different perspectives. The linguistic investigation provides interesting insights into current ELF practices and users' communicative and mediation strategies. Accommodative attitudes and actions of mutual intelligibility, typical of ELF encounters have also been confirmed.

Moreover, data provided in this study are extremely interesting from a pedagogical perspective, since they represent a vivid snapshot of what occurs in professional settings involving multicultural and multilingual components. On the one hand, the exploration of authentic materials may help to overcome stereotypical cultural depictions. On the other hand, the adaptation of spontaneous exchanges for the 
implementation of innovative teaching activities may be alternative to the employment of scripted dialogical exemplifications, often used for pedagogical and educational purposes.

First and foremost, the approach applied in this study may provide useful tools for the improvement of linguistic skills related to spoken abilities. The results have confirmed that the observation of real exchanges and the employment of mediation strategies are particularly useful in the promotion of the conscious use of learning tools embedded in the language learning process with the ultimate aim of (i) considering the impact of new socio-cultural dynamics emerging from the current migration flows and class composition on teacher education, (ii) implementing an ELF-aware pedagogical framework in plurilingual educational settings, and (iii) enhancing the development of learners' skills in intercultural communication.

At the same time, the analysis has also shown how difficult and challenging investigating (spontaneous) spoken discourse can be. Considered from this perspective, the training of interactional skills could be a useful pedagogical strategy applied to the planning of innovative materials and tasks in the plurilingual and multicultural classroom, from school to academic and professional contexts, especially in a prevailing ELF-oriented attitude and expanding scenario, in order to encourage learners to perform a successful and effective mediation role. In this sense, teachers should be properly trained in considering not only the pragmalinguistic processes involved in conversation (in terms of a correct semantic and pragmatic encoding and decoding of the linguistic message), but also paralinguistic and extralinguistic approaches and phonopragmatic habits deriving from different L1s and transferred by each speaker to his/her respective use of ELF.

Ultimately, teaching English adopting an ELF-oriented approach in multicultural contexts requires appropriate materials characterized by authenticity and reliability. Moreover, in the new multilingual and multicultural classrooms, learners' identities as well as socio-cultural dimensions are constantly challenged. First- and secondgeneration migrants, refugees and asylum-seekers, with all their socio-cultural and multilingual contribution and intake, are often involved in the educational process and dimension, where learners daily face and deal with the importance and the value of interacting in plurilingual and cross-cultural environments, developing corresponding effective mediation abilities and possessing suitable attitudes and strategies to cope with multicultural interferences and asymmetries.

Thus, the current development of English, in terms of World Englishes and English as a Lingua Franca, may not be disregarded. Moreover, learners are daily exposed to new forms of cross-cultural communication inside and outside the classroom. In this perspective, the results derived from the previous investigation underpin the emerging needs for a new approach in developing learners' language awareness, and their language skills, as well as in activating new processes for the acquisition of grammar and lexis. Course-books usually provide materials, tasks and activities on different cultures in a stereotyped way, disregarding the powerful value of training 
learners' mediation skills and communicative practices. The promotion of multilingualism and multicultural awareness in the ELT classroom along with an innovative approach to lesson planning may represent a first step at addressing the education challenges of the $21^{\text {st }}$ century.

\section{References}

Byram, M. (1995). Intercultural competence and mobility in multinational contexts. In D. Buttjes and M. Byram (Eds.), Mediating languages and cultures: Towards an intercultural theory of foreign language education (pp. 17-30). Clevedon: Multilingual Matters.

Carrell, P.L. (1983). Some issues in the role of schemata, or background knowledge, in second language comprehension. Reading in a Foreign Language, 1(2), 81-92.

Council of Europe. (2010). Guide for the development and implementation of curricula for plurilingual and intercultural education. Language Policy Unit. Strasbourg: Council of Europe.

Council of Europe. (2018). Common European framework of reference for languages: Learning, teaching, assessment. Companion Volume with new descriptors. Strasbourg: Council of Europe.

Edward, D. (1997). Discourse and cognition. London: Sage.

Guido, M. G. (2008). English as a lingua franca in cross-cultural immigration domains. Bern: Peter Lang.

Hynninen, N. (2011). The practice of 'mediation' in English as a lingua franca interaction. Journal of Pragmatics, 43(4), 965-977.

Kohler, M. (2015). Teachers as mediators in the foreign language classroom. Bristol: Multilingual Matters.

Lantolf, J.P. and Thorne, S. L. (2006). Sociocultural theory and the genesis of second language development. New York: Oxford University Press.

Mauranen, A. (2018). Conceptualising ELF. In J. Jenkins. M. J. Dewey, \& W. Baker (Eds.), The Routledge handbook of English as a lingua franca (pp. 7-24). London: Routledge.

Nespor, M. and Vogel, I. (1986). Prosodic phonology. Dordrecht: Foris Publications.

Roberts, C. and Sayers, P. (1987). Keeping the gate: How judgements are made in interethnic interviews. In K. Knapp, W. Enninger, \& A. Knapp-Potthoff (Eds.), Analyzing intercultural communication (pp. 111-135). Berlin: Mouton de Gruyter.

Searle, J.R. (1969). Speech acts. Cambridge: Cambridge University Press.

Searle, J.R. (1983). Intentionality: An essay in the philosophy of mind. Cambridge: Cambridge University Press.

Seidlhofer, B. (2011). Understanding English as a lingua franca. Oxford: Oxford University Press.

Selkirk, E. O. (1984). Phonology and syntax. The relation between sound and structure. Cambridge: MIT Press.

Sinclair, J. \& Coulthard, M. (1975). Towards an analysis of discourse: The English used by teachers and pupils. Oxford: Oxford University Press.

Sperti, S. (2017). Phonopragmatic Dimensions of ELF in Specialized Immigration Contexts. Centro di Ricerca sulle Lingue Franche nella Comunicazione Interculturale e Multimediale. Working Papers. Lecce: Università del Salento.

Widdowson, H.G. (1979). Explorations in applied linguistics. Oxford: Oxford University Press.

\section{Copyrights}

Copyright for this article is retained by the author(s), with first publication rights granted to the Journal.

This is an open-access article distributed under the terms and conditions of the Creative Commons Attribution license (CC BY-NC-ND) (http://creativecommons.org/licenses/by-nc-nd/4.0/). 